tricular tachycardia has previously been noted with serum concentrations of greater than $1000 \mu \mathrm{g} / \mathrm{l}$, and occasionally during intravenous administration. ${ }^{1}$ This patient developed ventricular tachycardia when a toxic serum flecainide concentration was achieved. It is likely that the ventricular tachycardia was a direct result of that serum concentration. The occurrence of ventricular tachycardia is of concern because of the association between sudden death and such arrhythmias.

Many cardiac units are using flecainide for first or second line treatment of supraventricular tachycardia, particularly in overt Wolff-Parkinson-White syndrome. Milk has not been reported to interfere with flecainide absorption in other children, but Wren and Campbell noted one patient (1 year old) who required a large oral dose $(22.5 \mathrm{mg} / \mathrm{kg} /$ day) to achieve a therapeutic serum concentration. ${ }^{6}$ It is possible that this may be a common phenomenon and merits further investigation. It becomes of particular importance in early infancy when the diet is milk alone. We recommend that milk fed infants on high doses of flecainide should have the dose reduced when milk is reduced or stopped-for example, during an attack of gastroenteritis, or when weaning. Close monitoring of plasma concentrations is likely to be helpful in the management of such patients.

\footnotetext{
References

1 Till JA, Rowland E, Ward DE. Treatment of refractory supraventricular arrhythmias with flecainide acetate. Arch Dis Child 1987;62:247-52.

2 Ward DE, Jones S, Shinebourne EA. Use of flecainide for refractory junctional tachycardias in children with the WolffParkinson-White Syndrome. Am J Cardiol 1986;57:787-90.

${ }^{3}$ Conrad GJ, Ober RE. Metabolism of flecainide. Am J Cardiol 1984;53:41-51B.

${ }^{4}$ Morganroth J, Horowitz LN. Flecainide: its proarrhythmic effect and expected changes on the surface electrocardiogram. Am J Cardiol 1984;53:89-94B.

5 Nathan AW, Hellestrand KJ, Bexton RS, Banim SO, Spurreil RAJ, Camm AJ. Proarrhythmic effects of the new antiarrhythmic agent flecainide acetate. Am Heart J 1984;107:222-8.

6 Wren C, Campbell RWF. The response of paediatric arrhythmias to intravenous and oral flecainide. Br Heart J 1987;57: $171-5$.
}

Correspondence to Dr RP Martin, Department of Paediatric Cardiology, Royal Hospital for Sick Children, St Michael's Hill, Bristol BS2 8BJ.

Accepted 3 January 1989

\title{
Phycomycotic abscesses in a preterm infant
}

\author{
P C NG AND P R F DEAR
}

Department of Paediatrics and Child Health, University of Leeds, St James's University Hospital

SUMMARY We report a case of neonatal infection with rhizopus, one of the Mucoraceae family. Human infection is rare but the mortality is high without prompt, correct treatment. The infant had a simultaneous candida septicaemia secondary to colonisation of a central venous line. Serial C reactive protein estimations are valuable in monitoring treatment.

Fungal skin abscesses (usually caused by Candida spp) are common among preterm babies, especially those receiving intensive care. ${ }^{1}$ We describe an abscess caused by an unusual pathogen, rhizopus, which, although well documented as a cause of serious infection in immunocompromised adults and children, ${ }^{2-4}$ has rarely been described in the newborn. ${ }^{3}$ The case also teaches an important lesson about the diagnosis and treatment of infection with multiple organisms.

\section{Case report}

A baby boy was born by emergency caesarean section (because of antepartum haemorrhage) at 26 weeks' gestation, weighing $1100 \mathrm{~g}$. Mild respiratory distress syndrome was diagnosed and the baby was initially managed in headbox oxygen. On the fourth day of life he required positive pressure ventilation and was transferred to the neonatal intensive care unit at this hospital.

On arrival his blood glucose concentration was 28 $\mathrm{mmol} / \mathrm{l}$, he had a metabolic acidosis, and was clinically dehydrated. Despite prompt rehydration he developed acute renal failure due to tubular necrosis. Peritoneal dialysis was needed for nine days until his renal function recovered. During dialysis coagulase negative staphylococcal peritonitis developed, but he was successfully treated with a combination of parenteral and intraperitoneal vancomycin. A Broviac catheter for venous access was sited in the internal jugular vein. 


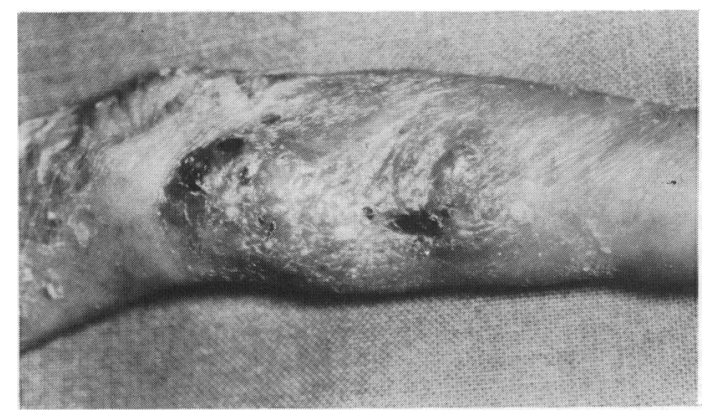

Figure Multiple abscesses on the baby's arm.

Two weeks after peritoneal dialysis and despite his seeming well, multiple and rapidly enlarging abscesses developed on his left forearm. They were erythematous, fluctuant and indurated, with necrotic areas at the centre (figure). Investigations to identify the infection were carried out, including serial $\mathrm{C}$ reactive protein estimations, venous and arterial blood cultures, urine culture and microscopy, and culture of the tracheal aspirate and of pus from the abscesses. Cloxacillin was given intravenously. All cultures were free from pathogens apart from the pus from the abscesses which, after 48 hours incubation on blood agar at $37^{\circ} \mathrm{C}$, grew a 'mucor-like' fungus. This was sensitive to amphotericin B and nystatin, but resistant to flucytosine, miconazole, econazole, and ketoconazole. Further culture of this organism on Sabouraud's medium and malt extract medium confirmed it to be a member of the Mucoraceae group. It was later identified as Rhizopus spp by the Regional Mycology Reference Laboratory. In view of the potential virulence of this particular family of fungi ${ }^{2-6}$ intravenous amphotericin B was started (despite the previous renal problem) in a dose of $0.25 \mathrm{mg} / \mathrm{kg} /$ day that was gradually increased to the full dose of $1 \mathrm{mg} / \mathrm{kg} /$ day over a week. The abscesses were surgically drained.

During the following week the baby became unwell with frequent attacks of apnoea and bradycardia that eventually required mechanical ventilation. The liver and spleen gradually enlarged, and the abscesses on the left forearm persisted. A skeletal survey did not show any focus of osteomyelitis, and echocardiography did not show any vegetation. Further investigations to find the cause of the infection were carried out. Microscopy and culture of the cerebrospinal fluid showed no pathogens. Three serial urine cultures contained a yeast, later identified as Candida parapsilosis, and both venous and arterial blood cultures grew the same yeast, which was sensitive to amphotericin B, nystatin, flucytosine, ketoconazole, miconazole, and econazole. We gave flucytosine $(100 \mathrm{mg} / \mathrm{kg} /$ day in four divided doses) and supplemented the antimycotic treatment with a dose of intravenous immunoglobulin $(200 \mathrm{mg} / \mathrm{kg})$. The baby showed an initial improvement, the $C$ reactive protein concentration falling from $153 \mathrm{mg} / \mathrm{l}$ to $78 \mathrm{mg} / \mathrm{l}$. Subsequently, however, the $\mathrm{C}$ reactive protein concentration increased to $270 \mathrm{mg} / \mathrm{l}$, which coincided with further deterioration. Further cultures were carried out, and the Broviac catheter was removed. Although urine cultures now grew no pathogens, blood cultures were still positive for candida and a profuse growth of Candida parapsilosis was obtained from the catheter tip. Clinical improvement was rapid thereafter, the $\mathrm{C}$ reactive protein concentration falling to $24 \mathrm{mg} / \mathrm{l}$ within 10 days.

After four weeks of treatment with full doses of amphotericin B and flucytosine, repeat venous and arterial blood cultures and urine cultures were all free of pathogens. Despite our fears of nephrotoxicity the baby showed no deterioration in renal function as judged by the plasma urea and creatinine concentrations. As is almost invariably the case with treatment with amphotericin B, the potassium intake had to be increased from $2 \mathrm{mmol} / \mathrm{kg} /$ day to 5 $\mathrm{mmol} / \mathrm{kg} /$ day to compensate for the increased tubular losses. The abscesses healed with minimal scarring and the baby continues in good health at the time of writing.

\section{Discussion}

Rhizopus, absidia, and mucor are a group of fungi belonging to the Mucoraceae family, and characterised by broad, branching, and non-septate hyphae. They grow naturally on a variety of organic substances such as bread, fruit, decaying vegetation, and carbohydrate rich substrates. Pathologically they represent an acute and frequently fulminant fungal infection with vascular invasion and tissue infarction as their hallmark. ${ }^{3-6}$

The liberal use of broad spectrum antibiotics and corticosteroids in neonatal practice undoubtedly encourages colonisation with opportunistic pathogens, and the frequent use of indwelling intravenous lines provides plenty of portals of entry. In our unit over $70 \%$ of the episodes of septicaemia are caused by coagulase negative staphylococci and we have noticed an increase in the incidence of fungal infections. Infection with rhizopus was nevertheless outside our experience. We presumed that the fungal abscesses developed at a 'tissued drip' site that was later covered with sterile dressing secured by adhesive tape. Most phycomycotic cellulitis 
occurs after previous injury to the tissues or necrosis, ${ }^{3-5}$ and the use of adhesive strapping ${ }^{35}$ has also been implicated. In debilitated patients, rhizopus infection of the lungs, gastrointestinal tract, ${ }^{3}$ sino-orbital region, ${ }^{2}$ brain, and $\operatorname{skin}^{4}{ }^{5}$ has been reported and the result is often fatal. Although purely cutaneous involvement unassociated with gangrenous cellulitis does seem to have a more favourable outcome, fatal cases have also been reported. ${ }^{2}$ The comparatively few reports of successfully treated cases ${ }^{2} 346$ have usually entailed extensive surgical resection in addition to the systemic use of amphotericin B. Our patient was treated with drainage of the abscesses and intravenous amphotericin B. Amphotericin B must be used, as this fungus is often resistant to the more commonly used antifungal agents, which in our case included miconazole, ketoconazole, econazole, and flucytosine. Because of its fulminant pathogenic nature with the ability to produce a rapidly progressive and often fatal gangrenous cellulitis, ${ }^{45}$ prompt treatment is always essential. Moreover, prolonged use of adhesive strapping on damaged tissue should also be avoided.

The candida septicaemia in this baby was almost certainly secondary to colonisation of the Broviac catheter, but despite the infusion of potent antifungal drugs through the line this focus was not eradicated. The persistent rise in the $C$ reactive protein concentration was a useful indicator of the presence of continuing infection, which was only eradicated after removal of the line.

\section{A new cardiomyopathy in girls}

\section{O IKEOGU}

\section{Department of Paediatrics, Mpilo Central Hospital, Bulawayo, Zimbabwe}

SUMMARY A type of cardiomyopathy characterised by isolated severe dilatation of the right heart with right ventricular hypertrophy and absence of cardiac arrhythmias is described in seven patients. Only girls were affected and all except one lived in rural Zimbabwe. Six were under 5 years old. This probably represents a new type of cardiomyopathy.

Cardiomyopathies are uncommon in childhood but all the types seen in the adult population have also been described in children. ${ }^{1}$ Their overall contribu-
We conclude that rhizopus infection must be considered as a cause of cutaneous abscess in the newborn and if confirmed must be treated without delay by surgical drainage and parenteral amphotericin $\mathrm{B}$. We have also shown the value of serial measurements of $C$ reactive protein concentrations in monitoring the treatment of systemic fungal infections. Central venous lines should be removed promptly if fungal septicaemia is suspected.

We thank the microbiology department at St James's University Hospital and the Regional Mycology Reference Laboratory at Leeds General Infirmary.

\section{References}

${ }^{1}$ Hensey OJ, Hart CA, Cooke RWI. Candida albicans skin abscesses. Arch Dis Child 1984;59:470-80.

2 Rosenberger RS, Burton CW, King JW. Case report-survival from sino-orbital mucormycosis due to rhizopus rhizopodiforms. Am J Med Sci 1983;286:25-30.

3 Dennis JE, Rhodes KH, Cooney DR, Roberts GD. Nosocomial rhizopus infection (zygomycosis) in children. J Pediatr 1980;96: $824-8$.

${ }^{4}$ Wilson CB, Siber GR, O'Brien TF. Phycomycotic gangrenous cellulitis. A report of 2 cases and a review of literature. Arch Surg 1976;111:532-8.

5 White CB, Barcia PJ, Bass JW. Neonatal zygomycotic necrotizing cellulitis. Pediatrics 1986;78:100-2.

6 Coetzee AS, De Bruin GF. Mucomycosis: case report and review. S Afr Med J 1974;48:2486-8.

Correspondence to Dr PRF Dear, St James's University Hospital, Beckett St, Leeds LS9 7TF.

Accepted 24 November 1988

tion to childhood cardiac disease remains largely unknown. This article describes a form of cardiomyopathy that has so far not been described in children.

\section{Patients and methods}

Seven girls between 3 months and 14 years of age were seen over a 22 month period in our paediatric cardiac clinic for assessment of unexplained cardiomegaly or heart failure. Six were under the age of 5 years. All had chest radiographs and a 12 lead electrocardiogram was available in five patients. 